Western University

Scholarship@Western

Robarts Imaging Publications

Robarts Research Institute

2016

\title{
Investigation of hippocampal substructures in focal temporal lobe epilepsy with and without hippocampal sclerosis at 7T.
}

Brendan G Santyr

Maged Goubran

Jonathan C Lau

Benjamin Y M Kwan

Fateme Salehi

See next page for additional authors

Follow this and additional works at: https://ir.lib.uwo.ca/robartspub

Part of the Bioimaging and Biomedical Optics Commons

Citation of this paper:

Santyr, Brendan G; Goubran, Maged; Lau, Jonathan C; Kwan, Benjamin Y M; Salehi, Fateme; Lee, Donald H; Mirsattari, Seyed M; Burneo, Jorge G; Steven, David A; Parrent, Andrew G; de Ribaupierre, Sandrine; Hammond, Robert R; Peters, Terry M; and Khan, Ali R, "Investigation of hippocampal substructures in focal temporal lobe epilepsy with and without hippocampal sclerosis at 7T." (2016). Robarts Imaging Publications. 16.

https://ir.lib.uwo.ca/robartspub/16 


\section{Authors}

Brendan G Santyr, Maged Goubran, Jonathan C Lau, Benjamin Y M Kwan, Fateme Salehi, Donald H Lee, Seyed M Mirsattari, Jorge G Burneo, David A Steven, Andrew G Parrent, Sandrine de Ribaupierre, Robert R Hammond, Terry M Peters, and Ali R Khan 


\section{TITLE}

Investigation of hippocampal sub-structures in focal temporal lobe epilepsy with and without hippocampal sclerosis at $7 \mathrm{~T}$.

\section{AUTHORS}

Brendan G. Santyr $(\mathrm{BSc})^{1}$, Maged Goubran $(\mathrm{PhD})^{1,2}$, Jonathan C. Lau (MD) ${ }^{3}$, Benjamin Y.M. Kwan (MD) $)^{1,2}$, Fateme Salehi (MD) ${ }^{1,2}$, Donald H. Lee (MD) ${ }^{1,2}$, Seyed M. Mirsattari $(\mathrm{MD})^{3}$, Jorge G. Burneo (MD) ${ }^{3}$, David A. Steven (MD) ${ }^{3}$, Andrew G. Parrent (MD) ${ }^{3}$, Sandrine de Ribaupierre (MD) ${ }^{3}$, Robert R. Hammond (MD) ${ }^{4}$, Terry M. Peters $(\mathrm{PhD})^{1,2}$, Ali R. Khan $(\mathrm{PhD})^{1,2}$

${ }^{1}$ Schulich School of Medicine and Dentistry - Western University, London, Ontario, Canada

${ }^{2}$ Departments of Medical Biophysics and Medical Imaging - Western University, London, Ontario, Canada

${ }^{3}$ Department of Clinical Neurological Sciences - London Health Sciences Centre, London, Ontario, Canada

${ }^{4}$ Department of Pathology and Laboratory Medicine - London Health Sciences Centre, London, Ontario, Canada

\section{CORRESPONDING AUTHOR}

Ali R. Khan

Departments of Medical Biophysics \& Medical Imaging Western University 
Robarts Research Institute

1151 Richmond St N

London, ON, Canada, N6A 5B7

e.alik@robarts.ca

t. $519.931 .5777(24280)$

\section{ACKNOWLEDGMENTS}

We would like to acknowledge Joe Gati and Trevor Szekeres for development and testing of the 7T MRI protocols.

\section{GRANT SUPPORT}

This work was supported by grants from CIHR (MOP 184807) and NSERC.

\section{RUNNING TITLE:}

Hippocampal sub-structures at 7T. 


\section{ABSTRACT}

Purpose: To provide a more detailed investigation of hippocampal subfields using ultrahigh field magnetic resonance imaging (MRI) for the identification of hippocampal pathologies in temporal lobe epilepsy.

Materials and Methods: Patients ( $\mathrm{N}=13)$ with drug-resistant TLE (9 with HS-no, 4 HS) and 20 age-matched healthy controls were scanned and compared using a 7T MRI protocol. Using a manual segmentation scheme to delineate hippocampal subfields, subfield-specific volume changes and apparent transverse relaxation rate $\left(R_{2}^{*}\right)$ were studied between the two groups. In addition, radiological patient assessment at 7T and clinical outcomes were correlated with measured subfield changes.

Results: Volumetry of the hippocampus at 7T in HS patients revealed significant ipsilateral subfield atrophy in CA1 and CA4DG. Volumetry also uncovered subfield atrophy in $33 \%$ of HS-no patients, which had not been detected using conventional imaging.

Conclusions: These preliminary findings indicate that hippocampal subfield volumetry assessed at 7T may be superior to conventional (1.5T) visual inspection by a neuroradiologist in the identification of hippocampal pathologies in TLE, however difficulty remains in identifying HS-no cases by imaging.

\section{KEY WORDS}

Ultra-high field MR; Hippocampal subfield; Volumetry; Transverse relaxation rate; Temporal lobe epilepsy 


\section{INTRODUCTION}

Temporal lobe epilepsy (TLE) is the most common form of adult focal epilepsy. For approximately $30 \%$ of TLE patients the condition is medically intractable (1), with surgical treatment as the standard of care. Surgical resection of the affected region of the brain aims to remove the seizure-inducing epileptogenic foci while excluding eloquent cortex and pathways, crucial for sensory, motor, and linguistic function. However, less than $50 \%$ of patients become seizure-free at 10 years after surgery (2), and there is a risk of deficit in language, auditory, and cognitive processes from damage to surrounding cortex and pathways (3).

The pathology of TLE most commonly manifests as hippocampal sclerosis (HS), characterized by neuronal loss and gliosis of specific subfields of the hippocampus and clinically by increased signal intensity on imaging $(4,5)$. Although lesions associated with the epileptogenic focus, like HS, can often be identified by MRI, many of the HS-no patients have unidentifiable lesions on conventional 1.5T MRI (6). In cases with identifiable HS, post-operative surgical outcomes are more favourable when compared to MRI-negative cases (4, 7-9). Furthermore, histopathological studies looking at subfield atrophy patterns in TLE patients with HS demonstrate that patients with more extensive subfield atrophy are more likely to become seizure free than those with more limited neuron loss $(4,8)$. Hence, having pre-operative knowledge pertaining to the extent and distribution of hippocampal neuronal loss may give insight into the prognosis of surgical candidates, potentially affecting surgical planning. 
The hippocampus is a compound temporal lobe structure of the brain traditionally divided into the hippocampal head, body, and tail. The hippocampal body comprises structurally and functionally distinct subregions (subfields) (10); the subiculum (SUB), ammon's horn (CA1-CA4), and dentate gyrus (DG). Histopathological studies have shown that disease processes selectively affect the different morphologically and functionally distinct subfields of the hippocampus $(11,12)$, for instance TLE has been characterized by loss of tissue in the CA1, DG, and global hippocampal atrophy (13-16). To study this further, Mueller et al. (2009) manually segmented the hippocampi of TLE patients to demonstrate the validity of using subfield volumetry in the detection of hippocampal atrophy at 4T MRI. In their study, regional hippocampal volume losses in CA1, CA3, and DG, with relative sparing of CA2 was detected in TLE patients with HS defined by visual assessment at $1.5 \mathrm{~T}$. Furthermore, the authors suggest the superiority of subfield volumetry over conventional hippocampal volumetry for the detection of hippocampal pathologies in TLE. This claim was moderately supported through the detection of hippocampal volume losses in 17\% of TLE patients without identified HS (Mueller et al., 2009).

Clinical 1.5T and 3.0T MRI scanners have limited ability to image the internal architecture of the hippocampus due to spatial resolution, contrast, and imaging constraints (18). Higher field strength MRI scanners provide a gain in intrinsic MR signal because of the linear relationship between the signal to noise ratio (SNR) and magnetic field strength. This increased SNR can also be exploited through imaging with increased resolution at 7T, which can consistently reveal hippocampal subfield morphometry at 
$300-500 \mu \mathrm{m}$ within a clinically acceptable imaging duration $(19,20)$. This advancement in imaging technology gives us the capability to study minute changes in hippocampal anatomy associated with TLE ideally suitable for clinical, in-vivo, and preoperative analysis.

Susceptibility weighted imaging (SWI) is an MRI technique that takes advantage of magnetic susceptibility differences between different tissues, for example blood, iron, and calcifications. One measure of SWI is the apparent transverse relaxation rate $\left(R_{2}^{*}\right)$. Previous work investigated the use of susceptibility related contrast for in vivo characterization of the hippocampal subfields at $7 \mathrm{~T}$ and found significant differences in $R_{2}^{*}$ across adjacent subfields (21). Therefore, this work suggests that quantitative maps based on SWI may be superior in sensitivity for the detection of abnormalities of specific hippocampal subfields than magnitude images and provides motivation for analysis of subfield composition in healthy and diseased populations (21).

The study of hippocampal subfield volumetry may be superior compared to conventional hippocampal volumetry for the detection of hippocampal pathologies in TLE. Furthermore, the identification of HS is clinically important in predicting surgical outcomes. In cases where gross HS is not identifiable in clinical 1.5T MRI, a more detailed analysis of hippocampal subfields using high field MRI may reveal areas of sclerosis. The effectiveness of ultra-high field magnetic resonance techniques in assessing and localizing abnormal structure of the temporal lobe was studied here. This work focused mainly on in-vivo detection of pathological TLE sub-types as the principle 
objective. This goal was accomplished through the development of a normative database of hippocampus structure using healthy volunteer 7T MRI scans. Using a manual segmentation scheme to delineate hippocampal subfields, this normative database was used to compare hippocampal subfield volume and $R_{2}^{*}$ between healthy controls and TLE patients. Subfield-specific changes in volume and $R_{2}^{*}$ were studied and radiological patient assessment at 7T was correlated with measured subfield changes and surgical outcomes.

\section{MATERIALS AND METHODS}

\section{Study Population}

Thirteen patients with drug-resistant TLE were recruited from the London Health Sciences Centre Comprehensive Epilepsy program, where they underwent continuous video-EEG monitoring followed by anterior temporal lobectomy. All recruited patients had preoperative investigations including neuropsychological testing and a 1.5T MRI with epilepsy protocol. Clinical data were collected for each patient, including age at surgery, gender, suspected epilepsy type, clinical 1.5T MRI reports, EEG reports, subdural electrode recording reports, clinical follow-up notes post resection and corresponding histopathology reports if applicable. This project was approved by the Office of Research and Ethics of Western University, and informed consent was obtained from all patients prior to their recruitment in the study. Exclusion criteria included patients with severe coexisting medical conditions and those unsuitable for MRI 
evaluation. Nine patients (mean age $31 \pm 12.57$, left TLE/right TLE 6/3, female/male 3/6) had normal-appearing hippocampi on their 1.5T MR examinations (HS-no) and 4 patients (mean age $36 \pm 12.78$, left TLE/right TLE 2/2, female/male 3/1) had evidence of HS on their 1.5T MR images in visual assessment by an experienced neuroradiologist (Table 1). The control population consisted of 20 healthy volunteers (mean age 31.2, female/male 10/10).

\section{MRI Acquisition}

Ultra-high field data were acquired on a $7 \mathrm{~T}$ neuroimaging optimized MRI scanner (Agilent, Santa Clara, CA, USA/ Siemens, Erlangen, Germany) using a 16-channel transmit- receive head coil array constructed in-house. The imaging sequences used for this study were multi-echo gradient-echo sequences with six echoes acquired and a 0.5 $\mathrm{mm}$ in-plane resolution $(\mathrm{TR}=40 \mathrm{~ms}, \mathrm{TE} 1=4.57 \mathrm{~ms}$, echo spacing=4.89 ms, flip

angle $=13, \mathrm{~N}=1$, matrix $=256 \times 360,80$ slices, slice thickness $=1.5 \mathrm{~mm}, \mathrm{FOV}=128$ x $180 \times 120 \mathrm{~mm}$, total time $=12 \mathrm{~min}$ ). Slices were acquired perpendicular to the long axis of the hippocampus in a coronal oblique orientation, in addition to a T1-weighted MPRAGE sequence $($ matrix $=256 \times 512 \times 172$, resolution $=0.58 \times 0.43 \times 1 \mathrm{~mm}$, scan time $=$ 5:42 min). These images were assigned randomized IDs assigned for visual interpretation by the neuroradiologists. We bias-corrected the images by employing the non-uniformity correction filter (N4) (22).

\section{Radiological Assessment}


High-resolution images acquired at 7 Tesla had mesial temporal internal architecture and mesial temporal size graded on a 4-point scale (normal, probably normal, possibly abnormal, and definitely abnormal) by an experienced neuroradiologist while blinded to clinical data. This data was used and further analyzed in our previous paper currently in submission (Kwan B, manuscript submitted). For the purpose of this study, if either mesial temporal internal architecture or mesial temporal size was abnormal it was designated hippocampal abnormality (HL). If both size and architecture were abnormal it was designated hippocampal sclerosis (HS). The criteria used for classification includes size differences (atrophy), abnormality in shape, and increased signal.

\section{Manual Marking of Hippocampal Subfields}

An ultra-high field 7T MRI protocol for manual hippocampal subfield delineation was adapted from the $4 \mathrm{~T}$ subfield delineation protocol proposed by (23) as well as the Duvernoy et al. (2005) atlas (10). This method for manual hippocampal subfield delineation was described in detail by Goubran et al., (2014) (Figure 1). Subfields were segmented in the body of the hippocampus only, defined as 6 slices just posterior to the head of the hippocampus. The following subregions were segmented in these 6 slices; Subiculum (Sub), Ammon's horn (CA1-CA3), and CA4+Dentate Gyrus (DG). The SUB is defined as the most medial border of the temporal cortex and its border with CA1 as a vertical line at the edge of the SUB touching the most medial border of the DG+CA4 region. The $\mathrm{CA} 1 / \mathrm{CA} 2$ boundary is defined as the point where a noticeable decrease in 
width of the CA1 subfield is observed. The overall shape of the hippocampal formation through the body slices was determined by the course of the outer boundary of the hippocampus and the hypointense line representing myelinated tissue in the strata moleculare/lacunosum (SLM). The opening of subfields into the globular region of the hippocampal formation defines the $\mathrm{CA} 3 / \mathrm{DG}+\mathrm{CA} 4$ border, which was specifically identified by the continuation of a clear consistent hypointense line representing the SLM of CA and dendrites of the molecular layer of DG, as described by (24).

With the manual segmentation protocol defined, it was implemented on 33 randomized subjects using the ITKsnap software (25). All images were visually inspected for accuracy and edited when necessary.

\section{Quantitative Measurements}

Using the FSL software the mean volume of each subfield was computed and used for subsequent analysis (26).

The apparent transverse relaxation rate, $R_{2}^{*}=\frac{1}{T_{2}^{*}}$, was calculated using a LevenbergMarquardt, least-squares fitting routine for non-linear equations. Specifically, the following mono- exponential decay function:

$$
S(T E)=S_{0} \cdot \exp \left(-T E \cdot R_{2}^{*}\right)
$$


was fit to the magnitude data on a voxel-by voxel basis. In Eq. (1), TE represents the echo time in ms and $S_{0}$ the signal intensity at $T E=50$. The mean $R_{2}^{*}$ over each subfield area was computed and used for subsequent analysis.

\section{Statistical Analysis}

For members of the control group, the right and left hippocampi were treated separately for the purpose of analysis. The subfield and whole hippocampal volume and $R_{2}^{*}$ data for each of the control hippocampi were averaged for comparison with the patient population. Patient volume and $R_{2}^{*}$ data were separated into ipsi- and contralateral designations. Multivariate analyses of covariance (MANCOVAs) were performed with ipsilateral subfield or whole hippocampal volumes as dependent variables. This analysis addressed the question of whether subfield volume depends on the group designation (HS, HS-no, control), when you control for age and intracranial volume (ICV). A Tukey post hoc analyses was completed for the MANCOVAs. This was similarly repeated for ipsilateral $R_{2}^{*}$ and their contralateral counterparts.

Normalized z-scores were computed using the control data to compare the severity of volume and $R_{2}^{*}$ deviations between different subfields. The volumes were first normalized for head size and converted into z-scores in the same manner outlined by Mueller et al., (2009). Analyses of variance (ANOVAs) and Tukey post hoc analyses were used to assess subfield atrophy. These z-scores were further used to identify subjectspecific patterns of change in volume or $R_{2}^{*}$. Additionally, the Pearson Correlation was 
used to compare the radiological assessment of mesial temporal internal architecture and mesial temporal size with the quantitative measures of subfield volume.

For patients that underwent surgical resection, outcomes were assessed using Engel classification (27). Its correlation with subfield volume and $R_{2}^{*}$ and radiological assessment was determined using the Pearson Correlation.

\section{RESULTS}

\section{Tesla Volumetry}

A significant ipsilateral volume group effect was revealed by MANCOVA [group: Wilks' Lambda $0.620 ; \mathrm{F}(10,88)=2.375 ; \mathrm{p}=0.015]$, indicating that there were differences between the HS, HS-no, and control groups for the CA1 ( $p=0.009)$, CA2+CA3 $(p=0.005)$, and CA4+DG $(p<0.001)$ ipsilateral subfields and whole hippocampal volume $(\mathrm{p}=0.001)$. Conversely, no significant contralateral volume group effects were revealed by MANCOVA, meaning contralateral subfields or whole hippocampal volumes were not different between the three groups. The Tukey post hoc analyses on the ipsilateral volume showed that the HS group had significantly smaller CA1 and CA4+DG subfields and a smaller whole hippocampus than controls $(p=0.001$, $\mathrm{p}<0.001, \mathrm{p}<0.001)$ and HS-no patients $(\mathrm{p}=0.013, \mathrm{p}<0.001, \mathrm{p}=0.001)$. There was a trend towards an ipsilateral volume loss in CA2+CA3. Additionally, the ipsilateral 
subfield and whole hippocampal volumes in the HS-no group were not different from those in controls (Table 2).

Likewise for $R_{2}^{*}$, a significant ipsilateral group effect was revealed by MANCOVA [group: Wilks' Lambda 0.603; F $(10,88)=2.531 ; \mathrm{p}=0.010$ ] for the CA4+DG subfield $(\mathrm{p}$ $=0.001)$ and and the whole hippocampal $R_{2}^{*}(\mathrm{p}=0.008)$ and none revealed contralaterally. Tukey post hoc analyses of the ipsilateral HS $R_{2}^{*}$ showed significantly lower $R_{2}^{*}$ in the CA4+DG subfield $(\mathrm{p}=0.001)$ and the whole hippocampus $(\mathrm{p}=0.029)$ compared to controls but not significantly lower than the HS-no group. Ipsilateral subfield and whole hippocampus $R_{2}^{*}$ in the HS-no group were not different from those in controls (Table 2).

After subfield-specific volume losses were found, an ANOVA was performed using the normalized subfield volume z-scores to identify the subfields that are most affected in TLE-HS (Figure 2). Firstly, the ANOVA revealed a significant ipsilateral subfield effect $[F(4,15)=6.07 ; p=0.004]$ for HS patients. This effect was limited to only the ipsilateral side and not the contralateral or HS-no patients. An ANOVA was similarily performed using the subfield $R_{2}^{*}$ z-scores and revealed no ipsilateral or contralateral subfield effects in either HS or HS-no patients. Post-hoc analyses on the normalized subfield volume zscores of HS patients revealed significantly smaller CA1 and CA4+DG z-scores compared to the Sub subfield. The whole hippocampus z-score on the sclerotic side was also significantly smaller than the z-score for the Sub subfield. The HS-no group showed 
no significant effect for ipsi- or contralateral subfield z-scores. This indicates a selective volume loss in these subfields (Figures 2, 3, and 4).

The normalized z-scores were used to quantify the subject-specific change in volume and $R_{2}^{*}$ relative to the healthy control group (Table 3). This uncovered volume loss in 3 of 9 HS-no patients. One patient (\#8) demonstrated evidence for severe subfield atrophy (zscore $\leq-2$ ) in a pattern resembling that uncovered for HS patients. This patient showed severe ipsilateral volume loss in the CA4+DG subfield and less severe losses $(\mathrm{z}$-score $\leq$ 1) in CA1 and CA2+CA3 (Figure 3 and 4). The ipsi- and contralateral whole hippocampal volume of this patient was also greater than one standard deviation from the mean of the HS-no group. The two other HS-no patients (patients \#9 and \#11) showed evidence for considerable contralateral CA2+CA3 subfield atrophy (z-score $\leq-1)$. These patients had ipsi- and contralateral whole hippocampal volumes within the normal range of all HS-no patients.

\section{Radiological Assessment}

The 7T images were assessed by an experienced radiologist looking specifically at mesial temporal internal architecture and mesial temporal size. This assessment was presented in depth in our previous paper currently in submission (Kwan B, manuscript submitted). Briefly, 7T MRI revealed structural lesions in 6 of 9 HS-no patients (Table 4). 
Quantitatively, Pearson Correlation was used to determine the relationship between the qualitative assessment at 7T and hippocampal subfield volumetry. The radiological assessment of mesial temporal size demonstrated greatest correlation with the normalized volume z-scores of the CA1 and CA4+DG subfields and the whole hippocampal label, the correlations were $-0.33,-0.39$, and -0.28 respectively. Similarly, the radiological assessment of mesial temporal internal architecture had greatest correlation with the same subfields and coefficients of $-0.22,-0.25,-0.30$. Although there appeared to be a trend, no significance was achieved for any of the correlations with $\mathrm{P}>0.05$.

\section{Surgical Outcomes}

Patients undergoing temporal lobe resection were monitored through follow-up to assess their seizure control following surgery. Engel classification was assigned to each patient following resection and a Pearson correlation was calculated to determine the relationship between hippocampal volume at $7 \mathrm{~T}$ and surgical outcomes. There was no significant correlation found between Engel classification and ipsilateral whole hippocampal and subfield volume and $R_{2}^{*}(\mathrm{p}>0.05)$ using a Pearson correlation. Furthermore, there was no significant correlation $(\mathrm{p}>0.05)$ between surgical outcomes and the radiographic assessment of hippocampal abnormality.

\section{DISCUSSION}


The higher spatial resolution provided by ultra-high-field magnetic resonance imaging (MRI) at 7 Tesla, allowed improved signal-to-noise ratio revealing internal sub-structures of the hippocampus, not visible at the resolution provided by 1.5 Tesla. This work aimed to use the increased resolution for in-vivo detection of pathological TLE sub-types for the better prediction of surgical outcomes. To this end, a normative database of hippocampal sub-structures was developed through the manual segmentation of healthy volunteer MRI scans at 7 Tesla. Using the normative database, pre-operative and patient-specific analyses were made, comparing volumetric and apparent transverse relaxation rate $\left(R_{2}^{*}\right)$ between patients with TLE and the normative database. These comparisons were made to assess and localize structural abnormalities in the hippocampal sub-structures of TLE patients. Characteristic atrophy patterns were found in HS patients in the ipsilateral CA1 and CA4+DG subfields, as well as ipsilateral whole hippocampal atrophy. Three of the HS-no patients demonstrated subfield atrophy (z-score <-1) using 7T volumetry. Furthermore, $67 \%$ of HS-no patients showed radiologist identifiable abnormality at 7T, indicating improved identification of abnormality at higher field strength. The novel preoperative imaging techniques utilized in this project may help predict long-term surgical outcomes by identifying sclerotic and non-sclerotic sub-types of TLE prior to surgery. However, in this study the surgical outcomes were followed and no significant correlation was found.

Increased resolution and improved signal-to-noise at 7 Tesla allowed the marking of hippocampal subfields in healthy controls and TLE patients with and without HS. Attempts at a universal hippocampal segmentation protocol have been made $(28,29)$, 
however still debated. At the time of this study there existed no agreed upon segmentation protocol and thus a manual segmentation protocol was employed. The manual marking scheme used was based on details of internal structure of the hippocampus visible on high resolution T2-weighted images at 7T. Features were chosen that were reliably identifiable even in cases of severe sclerosis. As Mueller et. al. (2009) uncovered, there is preservation of internal architecture at higher field strengths, particularly 4T. A similar preservation of architecture was found at $7 \mathrm{~T}$ in HS patents, despite one of the main radiographic criteria for HS in clinical 1.5T MRI is signal abnormality and loss of the internal architecture. Although the appeal of using 7T MRI is the higher resolution it offers, it is still not sufficient to reliably and accurately identify the boundaries of each of the hippocampal subfield. Therefore, similar to Mueller et. at. (2009), reliable hippocampal landmarks in addition to radiographic landmarks were used to designate the hippocampal subfields.

Volumetry of the hippocampus at $7 \mathrm{~T}$ in HS patients revealed a characteristic pattern of significant global ipsilateral hippocampal atrophy, restricted subfield losses in CA1 and CA4+DG, and no significant contralateral volume losses. These results confirm those that have been found at 3T (30) and 4T (17), histological studies (31), as well as combined MRI-histology investigations (5) for patients with medically intractable TLE. The same marking scheme was used to assess the $R_{2}^{*}$ signal within the hippocampal subfields of HS, HS-no, and control groups. Although some significant subfield reductions in $R_{2}^{*}$ were measured between HS and the HS-no group, it was not possible to draw conclusions 
about the $R_{2}^{*}$ signal changes in TLE at 7T from this data without significant differences form the control group.

Not only did volumetry reveal the expected pattern of atrophy in this study but it was observed for each subject clinically identified as having HS, therefore confirming the clinical designation of HS. However, of note is the ability of 7T volumetry to go beyond the clinical designation of sclerosis and identify additional cases of sclerosis in $33 \%$ of HS-no patients in this study. Similar to the HS patients, who were characterized in this study by CA1, CA4+DG, and trending CA2+CA3 subfield atrophy, one of the HS-no patients (patient \#8) demonstrated a near identical pattern of atrophy. This may represent a patient with less severe HS, whose diagnosis of HS was missed on 1.5T clinical MRI. Therefore, the resolution provided by 7T MRI may provide novel information about TLE pathology not available by conventional imaging. The other two HS-no patients displaying subfield atrophy (patients \#9 and \#11), had volume losses mainly limited to the contralateral CA2+CA3 subfield. This difference in atrophy pattern between HS patients and HS-no patients suggests that patients with MRI-negative epilepsy isn't just a less advanced form of HS TLE but is instead a different type of TLE and is a testament to the heterogeneity of cases of MRI-negative epilepsy (6).

For this study, patients were initially classified as having HS or HS-no TLE clinically at 1.5T. Subsequently, following preoperative imaging at $7 \mathrm{~T}$, an experienced radiologist judged the scans on the basis of mesial temporal internal architecture and mesial temporal size. Blinded to the patient's initial classification, at 7T the radiologist confirmed 
abnormal internal architecture and size corresponding to clinically classified HS patients. Patients determined to have abnormal architecture or size at $7 \mathrm{~T}$ also had lower quantitative volumes in a pattern similar to that revealed by volumetry, selective losses in CA1, CA4+DG, and the whole hippocampus label. However, the correlation was not significant. Although the superiority $7 \mathrm{~T}$ volumetry compared to conventional $1.5 \mathrm{~T}$ visual assessment hasn't been confirmed in this study, our previous work (Kwan B, manuscript submitted) holds promise in demonstrating that radiological assessment at $7 \mathrm{~T}$ may be superior to conventional visual inspection by a neuroradiologist in the identification of hippocampal pathologies in TLE. Interestingly, the visual inspection at $7 \mathrm{~T}$ detected abnormality in more cases of HS-no than volumetry. Visual inspection looked at features of size difference (atrophy), abnormality in shape, and increased signal, where as volumetry measured differences in subfield volume and $R_{2}^{*}$. Therefore, subfield-specific changes in shape or intensity may provide a fruitful area for further study.

This study is limited by the inclusion of only thirteen TLE patients and thus the results must only be taken as preliminary. Secondly, although there are gains in intrinsic MR signal provided by higher field strength MRI scanners which result in improvements in spatial resolution, the boundaries between many subfields can only be defined microscopically, and thus the definitions here instead rely on geometric rules. Validation of the subfield marking scheme using neuronal cell counts on the resected tissue is considered future directions for improving the impact of this work. Currently only ten of thirteen patients have undergone surgery and resection. Of this subset, HS status has been confirmed but subfield-specific cell counts and correlation analysis still remains to be 
performed. A final limitation worth mentioning, similar to that which affected Mueller et. al. (2009), is that the hippocampal subfields were only marked in a small region of the anterior body, thus potentially missing atrophic changes restricted to the posterior body/tail and head of the hippocampus. Furthermore, there may be benefit in posterior atrophic changes and comparing these changes to those seen in the anterior body as a method of prognostication prior to surgery. It has been shown through histopathological studies that in TLE patients with atrophy restricted to the anterior hippocampus are more likely to benefit from surgery than those with diffuse atrophy (13).

In conclusion in these preliminary findings, $7 \mathrm{~T}$ volumetry demonstrated hippocampal atrophy patterns in excellent agreement with the typical patterns of atrophy identified in histological studies and studies at lower field strengths. Subfield volumetry also uncovered hippocampal subfield volume losses in $33 \%$ of HS-no patients, which had not been identified conventionally. This study suggests that hippocampal subfield volumetry or routine clinical radiological assessment by visual inspection at $7 \mathrm{~T}$ may be superior to conventional visual inspection by a neuroradiologist in the identification of hippocampal pathologies in TLE, however there remains difficulty in identifying HS-no cases by imaging. 


\section{REFERENCES}

1. Engel J: Surgery for seizures. N Engl J Med 1996; 334:647-652.

2. de Tisi J, Bell GS, Peacock JL, et al.: The long-term outcome of adult epilepsy surgery, patterns of seizure remission, and relapse: a cohort study. Lancet (London, England) 2011; 378:1388-95.

3. Vadera S, Kshettry VR, Klaas P, Bingaman W: Seizure-free and neuropsychological outcomes after temporal lobectomy with amygdalohippocampectomy in pediatric patients with hippocampal sclerosis. J Neurosurg Pediatr 2012; 10:103-7.

4. Blümcke I, Pauli E, Clusmann H, et al.: A new clinico-pathological classification system for mesial temporal sclerosis. Acta Neuropathol 2007; 113:235-44.

5. Goubran M, Bernhardt BC, Cantor-Rivera D, et al.: In vivo MRI signatures of hippocampal subfield pathology in intractable epilepsy. Hum Brain Mapp 2016; $37: 1103-19$.

6. Bernasconi A, Bernasconi N, Bernhardt BC, Schrader D: Advances in MRI for “cryptogenic” epilepsies. Nat Rev Neurol 2011; 7:99-108.

7. Mathern GW, Babb TL, Vickrey BG, Melendez M, Pretorius JK: The clinicalpathogenic mechanisms of hippocampal neuron loss and surgical outcomes in temporal lobe epilepsy. Brain 1995; 118 ( Pt 1:105-118.

8. De Lanerolle NC, Kim JH, Williamson A, et al.: A retrospective analysis of hippocampal pathology in human temporal lobe epilepsy: Evidence for distinctive patient subcategories. Epilepsia 2003; 44:677-687.

9. Thom M, Liagkouras I, Elliot KJ, et al.: Reliability of patterns of hippocampal sclerosis as predictors of postsurgical outcome. Epilepsia 2010; 51:1801-8. 
10. Duvernoy HM: The Human Hippocampus. Functional Anatomy, Vascularization and Serial Sections With MRI. Springer Verlag, Berlin, Heidelberg, New York 2005; 3rd.

11. West M., Coleman P., Flood D., Troncoso J.: Differences in the pattern of hippocampal neuronal loss in normal ageing and Alzheimer's disease. Lancet 1994; 344:769-772.

12. Lucassen PJ, Heine VM, Muller MB, et al.: Stress, depression and hippocampal apoptosis. CNS Neurol Disord Drug Targets 2006; 5:531-46.

13. Babb TL, Brown WJ, Pretorius J, Davenport C, Lieb JP, Crandall PH: Temporal lobe volumetric cell densities in temporal lobe epilepsy. Epilepsia 1984; 25:729-40.

14. Dam a M: Epilepsy and neuron loss in the hippocampus. Epilepsia 1980; 21:617629.

15. Bruton CJ: No TitleThe Neuropathology of Temporal Lobe Epilepsy. Oxford Univ Press Oxford 1988.

16. Wyler AR, Dohan FC, Schweitzer JB BA: A grading system for mesial temporal pathology (hippocampal sclerosis) from anterior temporal lobectomy. J Epilepsy 1992; $5: 220-225$.

17. Mueller SG, Laxer KD, Barakos J, Cheong I, Garcia P, Weiner MW: Subfield atrophy pattern in temporal lobe epilepsy with and without mesial sclerosis detected by high-resolution MRI at 4 Tesla: Preliminary results. Epilepsia 2009; 50:1474-1483. 18. Balchandani P, Naidich TP: Ultra-High-Field MR Neuroimaging. AJNR Am J Neuroradiol 2015; 36:1204-15.

19. Prudent V, Kumar A, Liu S, Wiggins G, Malaspina D, Gonen O: Human hippocampal subfields in young adults at 7.0 T: feasibility of imaging. Radiology 2010; 
254:900-6.

20. Ali R, Goubran M, Choudhri O, Zeineh MM: Seven-Tesla MRI and neuroimaging biomarkers for Alzheimer's disease. Neurosurg Focus 2015; 39:E4.

21. Goubran M, Rudko D a., Santyr B, et al.: In vivo normative atlas of the hippocampal subfields using multi-echo susceptibility imaging at 7 Tesla. Hum Brain Mapp 2014; $35: 3588-3601$.

22. Tustison NJ, Avants BB, Cook PA, et al.: N4ITK: improved N3 bias correction. IEEE Trans Med Imaging 2010; 29:1310-20.

23. Mueller SG, Stables L, Du AT, et al.: Measurement of hippocampal subfields and age-related changes with high resolution MRI at 4T. Neurobiol Aging 2007; 28:719-26. 24. Wisse LEM, Gerritsen L, Zwanenburg JJM, et al.: Subfields of the hippocampal formation at 7T MRI: In vivo volumetric assessment. Neuroimage 2012; 61:1043-1049. 25. Yushkevich PA, Piven J, Hazlett HC, et al.: User-guided 3D active contour segmentation of anatomical structures: significantly improved efficiency and reliability. Neuroimage 2006; 31:1116-28.

26. Woolrich MW, Jbabdi S, Patenaude B, et al.: Bayesian analysis of neuroimaging data in FSL. Neuroimage 2009; 45(1 Suppl):S173-86.

27. Wieser HG, Blume WT, Fish D, et al.: ILAE Commission Report. Proposal for a new classification of outcome with respect to epileptic seizures following epilepsy surgery. Epilepsia 2001; 42:282-6.

28. Kulaga-Yoskovitz J, Bernhardt BC, Hong S-JJ, et al.: Multi-contrast submillimetric 3 Tesla hippocampal subfield segmentation protocol and dataset. Sci Data 2015; 2:150059. 29. Yushkevich P a., Amaral RSC, Augustinack JC, et al.: Quantitative comparison of 21 
protocols for labeling hippocampal subfields and parahippocampal subregions in in vivo MRI: Towards a harmonized segmentation protocol. Neuroimage 2015.

30. Schoene-Bake J-C, Keller SS, Niehusmann P, et al.: In vivo mapping of hippocampal subfields in mesial temporal lobe epilepsy: relation to histopathology. Hum Brain Mapp $2014 ; 35: 4718-28$.

31. Steve TA, Jirsch JD, Gross DW: Quantification of subfield pathology in hippocampal sclerosis: a systematic review and meta-analysis. Epilepsy Res 2014; 108:1279-85. 


\section{TABLES}

Table 1. Patient Characteristics.

\begin{tabular}{|c|c|c|c|c|c|c|c|c|c|}
\hline Patient Number & $\begin{array}{l}\text { Age at } \\
\text { surgery }\end{array}$ & Gender & $\begin{array}{l}\text { Hemisphere of } \\
\text { Seizure Onset }\end{array}$ & $\begin{array}{c}\text { Age of seizure } \\
\text { onset }\end{array}$ & 1.5T MR Findings & $\begin{array}{c}\text { 1.5T MR } \\
\text { Designation }\end{array}$ & Hippocampal pathology & $\begin{array}{l}\text { Follow up duration } \\
\text { (Months) }\end{array}$ & $\begin{array}{c}\text { Postsurgical } \\
\text { outcome }\end{array}$ \\
\hline 1 & 20 & $\mathrm{M}$ & $\overline{\mathrm{L}}$ & 3 & $\begin{array}{l}\text { L HS, L occipital } \\
\text { lobe } \\
\text { encephalomalacia } \\
\text { (remote) }\end{array}$ & L HS & $\begin{array}{l}\text { Gliosis (temporal } \\
\text { neocortex and } \\
\text { amygdala), mesial } \\
\text { temporal sclerosis } \\
\text { (hippocampus) }\end{array}$ & 35 & I \\
\hline 2 & 32 & $\mathrm{~F}$ & $\mathrm{~L}$ & 19 & L HS & L HS & $\begin{array}{l}\text { Gliosis (temporal } \\
\text { neocortex), mesial } \\
\text { temporal sclerosis }\end{array}$ & 27 & I \\
\hline 3 & 49 & $\mathrm{~F}$ & $\mathrm{R}$ & 22 & $\begin{array}{l}\text { R HS, L } \\
\text { periventricular } \\
\text { heterotopia }\end{array}$ & R HS & $\begin{array}{l}\text { Gliosis (temporal } \\
\text { neocortex), mesial } \\
\text { temporal sclerosis }\end{array}$ & 23 & II \\
\hline 4 & 45 & $\mathrm{~F}$ & $\mathrm{R}$ & 3 & R HS & R HS & $\begin{array}{l}\text { Gliosis (temporal lobe), } \\
\text { mesial temporal sclerosis }\end{array}$ & 39 & I \\
\hline 5 & 18 & M & $\mathrm{R}$ & 14 & Possible R HS & HS-no & $\begin{array}{l}\text { Gliosis (temporal } \\
\text { neocortex, hippocampus } \\
\text { and amygdala) }\end{array}$ & 36 & I \\
\hline 6 & 24 & M & $\mathrm{L}$ & & Normal & HS-no & Normal & 7 & I \\
\hline 7 & NA & M & $\mathrm{L}$ & 26 & $\begin{array}{l}\text { Temporal } \\
\text { pole/gyrus rectus is } \\
\text { slightly smaller on } \\
\text { the left }\end{array}$ & HS-no & No resection & NA & NA \\
\hline 8 & 50 & $\mathrm{~F}$ & $\mathrm{~L}$ & 47 & $\begin{array}{l}\text { Equivocal changes } \\
\text { inferomedially in } \\
\text { the left temporal } \\
\text { pole }\end{array}$ & HS-no & $\begin{array}{l}\text { Gliosis (temporal } \\
\text { neocortex, amygdala, } \\
\text { and hippocampus) }\end{array}$ & 36 & IV \\
\hline 9 & 42 & $\mathrm{~F}$ & $\mathrm{R}$ & 8 & $\begin{array}{l}\text { Slightly increased } \\
\text { T2 signal in the } \\
\text { right hippocampus }\end{array}$ & HS-no & $\begin{array}{l}\text { Gliosis (temporal } \\
\text { neocortex, amygdala, } \\
\text { and hippocampus) }\end{array}$ & 17 & I \\
\hline 10 & NA & M & $\mathrm{L}$ & 18 & $\begin{array}{l}\text { Bifrontal } \\
\text { periventricular } \\
\text { heterotopia }\end{array}$ & HS-no & No resection & NA & NA \\
\hline 11 & 31 & M & $\mathrm{R}$ & 28 & Normal & HS-no & $\begin{array}{l}\text { Mild cortical dysplasia, } \\
\text { mild gliosis (temporal } \\
\text { neocortex) }\end{array}$ & 18 & I \\
\hline 12 & 23 & M & $\mathrm{L}$ & 18 & Normal & HS-no & $\begin{array}{l}\text { Gliosis (temporal } \\
\text { neocortex, amygdala, } \\
\text { and hippocampus), focal } \\
\text { cortical dysplasia type Ia } \\
\text { (temporal neocortex) }\end{array}$ & 18 & I \\
\hline 13 & NA & $\mathrm{F}$ & $\mathrm{L}$ & 44 & Normal & HS-no & No resection & NA & NA \\
\hline
\end{tabular}

R, right; L, left; HS, hippocampal sclerosis; MR, magnetic resonance; NA, not

applicable. Post-surgical outcome classification; according to Engel's classification of

postoperative outcomes. 
Table 2: Mean and (SD) of subfield and total hippocampal volumes and $R_{2}^{*}$.

\begin{tabular}{|c|l|c|c|c|c|c|}
\hline & & Controls, $\mathrm{n}=20$ & \multicolumn{2}{|c|}{ HS, $\mathrm{n}=4$} & \multicolumn{2}{c|}{ HS-no, $\mathrm{n}=9$} \\
\hline \multirow{5}{*}{ Volume } & & Mean left and right & Ipsilateral & Contralateral & Ipsilateral & Contraleral \\
\hline \multirow{5}{*}{$R_{2}^{*}$} & Sub & $115.8(30.8)$ & $115.3(11.3)$ & $139.8(32.2)$ & $121.2(30.2)$ & $117.5(31.5)$ \\
\cline { 2 - 7 } & CA1 & $131.6(26.5)$ & $93.5(25.6) \ddagger$ & $132.4(50.2)$ & $132.9(22.0)$ & $142.0(26.2)$ \\
\cline { 2 - 7 } & CA2+CA3 & $36.4(6.9)$ & $30.3(1.3)$ & $39.2(11.0)$ & $37.7(7.3)$ & $39.7(10.3)$ \\
\cline { 2 - 7 } & CA4+DG & $112.7(21.9)$ & $79.9(9.8) \ddagger$ & $94.9(20.2)$ & $122.2(21.5)$ & $120.2(22.8)$ \\
\cline { 2 - 7 } & WholeHipp & $1917.5(345.6)$ & $1367.8(119.1) \ddagger$ & $1822.1(356.6)$ & $2028.8(246.8)$ & $2076.3(322.0)$ \\
\hline \multirow{5}{*}{} & Sub & $33.0(4.4)$ & $29.6(3.2)$ & $34.1(4.6)$ & $33.7(3.5)$ & $32.1(3.4)$ \\
\cline { 2 - 7 } & CA1 & $26.3(3.2)$ & $24.4(5.1)$ & $26.9(4.6)$ & $26.3(3.3)$ & $27.0(2.5)$ \\
\cline { 2 - 7 } & CA2+CA3 & $30.1(4.5)$ & $28.2(6.8)$ & $32.5(7.7)$ & $31.5(3.8)$ & $30.9(4.0)$ \\
\cline { 2 - 7 } & CA4+DG & $31.0(4.3)$ & $22.4(4.1) \dagger$ & $31.0(3.3)$ & $28.2(5.0)$ & $31.0(2.6)$ \\
\cline { 2 - 7 } & WholeHipp & $29.0(2.0)$ & $26.0(3.5) \dagger$ & $28.7(2.8)$ & $28.7(2.4)$ & $29.7(2.0)$ \\
\hline
\end{tabular}

Units of subfield volume are in $\mathrm{mm}^{3}$. SD, standard deviation; HS, hippocampal sclerosis.

$\dagger \mathrm{p}<0.05$ compared to controls only.

$\$ \mathrm{p}<0.05$ compared to both HS-no and control groups.

Table 3: Normalized ipsilateral z-scores for volume.

\begin{tabular}{|c|c|c|c|c|c|c|}
\hline \multirow[b]{2}{*}{$\begin{array}{l}\text { Patient } \\
\text { Number }\end{array}$} & \multirow[b]{2}{*}{$\begin{array}{c}1.5 \mathrm{~T} \mathrm{MR} \\
\text { Designation }\end{array}$} & \multicolumn{5}{|c|}{ Hippocampal Subfield } \\
\hline & & Sub & CA1 & $\mathrm{CA} 2+\mathrm{CA} 3$ & CA4+DG & WholeHipp \\
\hline 1 & L HS & -0.44 & -1.82 & -0.76 & -2.10 & -1.58 \\
\hline 2 & L HS & 0.02 & -2.18 & -0.70 & -1.29 & -2.08 \\
\hline 3 & R HS & 0.46 & -0.02 & -0.96 & -1.05 & -1.31 \\
\hline 4 & R HS & -0.11 & -1.73 & -1.12 & -1.55 & -1.39 \\
\hline 5 & HS-no & 0.27 & 0.85 & 2.02 & 0.54 & 1.00 \\
\hline 6 & HS-no & -1.12 & 0.40 & 0.68 & 0.93 & -0.13 \\
\hline 7 & HS-no & 0.02 & -0.32 & 1.04 & 0.95 & -0.05 \\
\hline 8 & HS-no & -0.55 & -1.22 & -1.60 & -2.01 & -0.65 \\
\hline 9 & HS-no & 1.10 & -0.06 & -0.88 & 0.58 & 0.39 \\
\hline 10 & HS-no & -0.14 & -0.95 & 0.15 & 0.05 & 0.66 \\
\hline 11 & HS-no & 0.45 & 0.40 & -0.14 & 0.66 & 0.84 \\
\hline 12 & HS-no & -0.58 & -0.09 & 0.63 & 1.33 & -0.56 \\
\hline 13 & HS-no & 2.14 & 1.41 & -0.20 & 0.88 & 1.40 \\
\hline
\end{tabular}

HS, hippocampal sclerosis; MR, magnetic resonance.

Table 4: Radiological assessment at 7T. 


\begin{tabular}{|c|c|c|c|c|}
\hline \multirow{2}{*}{$\begin{array}{l}\text { Patient } \\
\text { Number }\end{array}$} & \multirow{2}{*}{$\begin{array}{c}1.5 \mathrm{~T} \mathrm{MR} \\
\text { Designation }\end{array}$} & \multirow{2}{*}{$\begin{array}{c}\text { 7T MR } \\
\text { Designation }\end{array}$} & \multicolumn{2}{|c|}{7 Tesla MRI (radiological assesment) } \\
\hline & & & $\begin{array}{c}\text { Mesial Temporal } \\
\text { Internal Architecture }\end{array}$ & Mesial Temporal Size \\
\hline 1 & L HS & L HS & L definitely abnormal & L definitely abnormal \\
\hline 2 & L HS & L HS & L definitely abnormal & L definitely abnormal \\
\hline 3 & R HS & R HS, L HL & R possibly abnormal & normal \\
\hline 4 & R HS & R HS & R possibly abnormal & R possibly abnormal \\
\hline 5 & HS-no & L HS & L possibly abnormal & L possibly abnormal \\
\hline 6 & HS-no & HS-no & R probably normal & normal \\
\hline 7 & HS-no & R HS & $\mathrm{R}$ possibly abnormal & R possibly abnormal \\
\hline 8 & HS-no & L HL & normal & L possibly abnormal \\
\hline 9 & HS-no & HS-no & normal & normal \\
\hline 10 & HS-no & R HL & $\mathrm{R}$ possibly abnormal & normal \\
\hline 11 & HS-no & L HS & L possibly abnormal & L possibly abnormal \\
\hline 12 & HS-no & HS-no & $\begin{array}{l}\mathrm{R}+\mathrm{L} \text { probably } \\
\text { normal }\end{array}$ & $\begin{array}{l}\mathrm{R}+\mathrm{L} \text { probably } \\
\text { normal }\end{array}$ \\
\hline 13 & HS-no & R HL, L HL & R possibly abnormal & L possibly abnormal \\
\hline
\end{tabular}

R, right; L, left; MR, magnetic resonance; HS, hippocampal sclerosis; HL, hippocampal abnormality (if either mesial temporal internal architecture or mesial temporal size is abnormal). 


\section{FIGURE LEGENDS}

Figure 1: Segmentation scheme for manual marking of subfields. The scheme used was based on details of internal structure of the hippocampus visible on high resolution T2weighted images at 7T. A typical example of hippocampal subfield marking on in vivo T2-weighted 7T coronal images of a control subject. CA2-3, CA2-CA3 transition zone; CA4\&DG, CA4 and dentate gyrus; Sub, subiculum. Numbers 1-6 represents each $0.5 \mathrm{~mm}$ slice of the hippocampal body that was segmented. No. 1 represents the most anterior slice and No. 6 the most posterior slice.

Figure 2: Mean and standard deviation of (A) volume and (B) $R_{2}^{*}$ subfield z-scores for HS (blue) and HS-no (red) patients. *Indicates p < 0.05 compared to Sub. Sub, subiculum; WholeHipp, whole hippocampus label.

Figure 3: Line plots demonstrating subject-specific patterns of ipsilateral atrophy. Zscores were calculated and plotted with respect to each subfield for every patient and grouped by 1.5T MRI designation. The single HS-no patient (patient \# 8) demonstrating an atrophy pattern similar to those designated HS was grouped separately. Sub, subiculum; WholeHipp, whole hippocampus label.

Figure 4: High resolution in vivo 7T T2-weighted representative coronal images of the hippocampal body demonstrating patterns of sclerotic change, with arrows indicating atrophy: (A) 33-year-old control. (B) 20-year-old HS patient with CA1 and CA4+DG volume loss and relative sparing of the other subfields. (C) 32-year-old HS patient with severe CA1 thinning and lesser CA4+DG atrophy with relative sparing of the other subfields. (D) 50-year-old HS-no patient with CA1 and CA4+DG volume loss (patient \#8). 


\section{FIGURES}
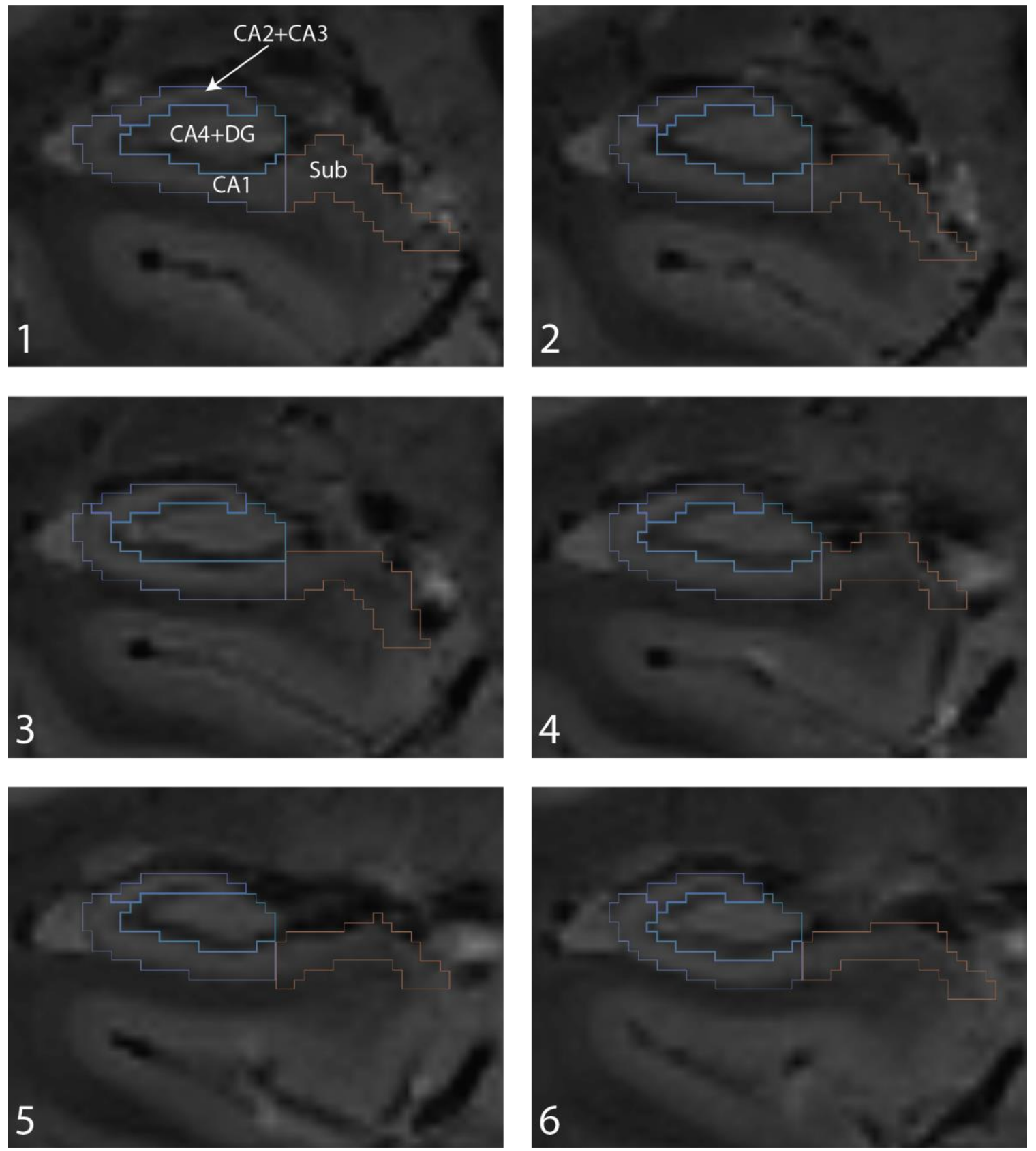

Figure 1: Segmentation scheme for manual marking of subfields. The scheme used was based on details of internal structure of the hippocampus visible on high resolution T2weighted images at 7T. A typical example of hippocampal subfield marking on in vivo T2-weighted 7T coronal images of a control subject. CA2-3, CA2-CA3 transition zone; 
CA4\&DG, CA4 and dentate gyrus; Sub, subiculum. Numbers 1-6 represents each $0.5 \mathrm{~mm}$ slice of the hippocampal body that was segmented. No. 1 represents the most anterior slice and No. 6 the most posterior slice.
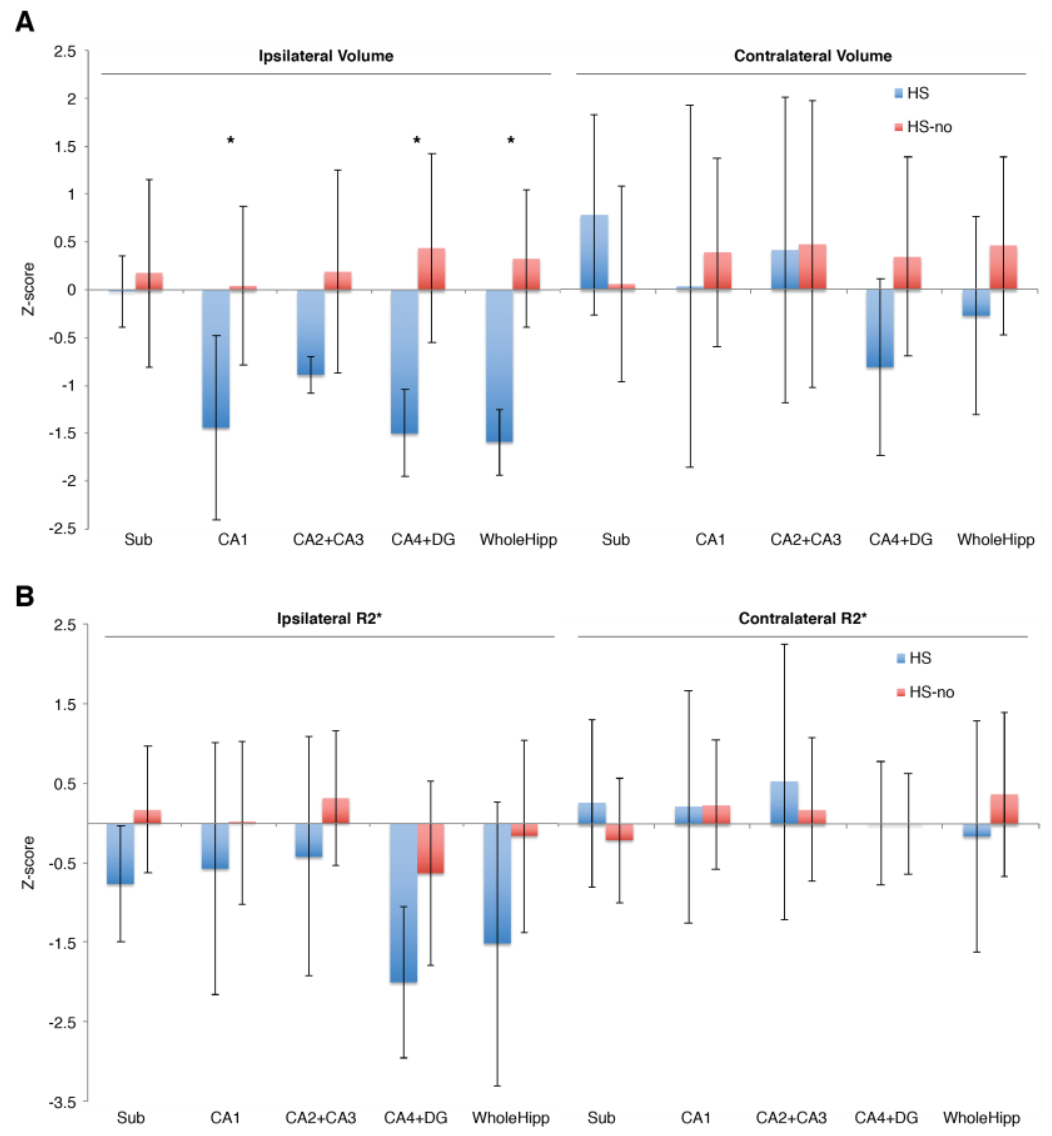

Figure 2: Mean and standard deviation of (A) volume and (B) $R_{2}^{*}$ subfield z-scores for HS (blue) and HS-no (red) patients. *Indicates p < 0.05 compared to Sub. Sub, subiculum; WholeHipp, whole hippocampus label. 


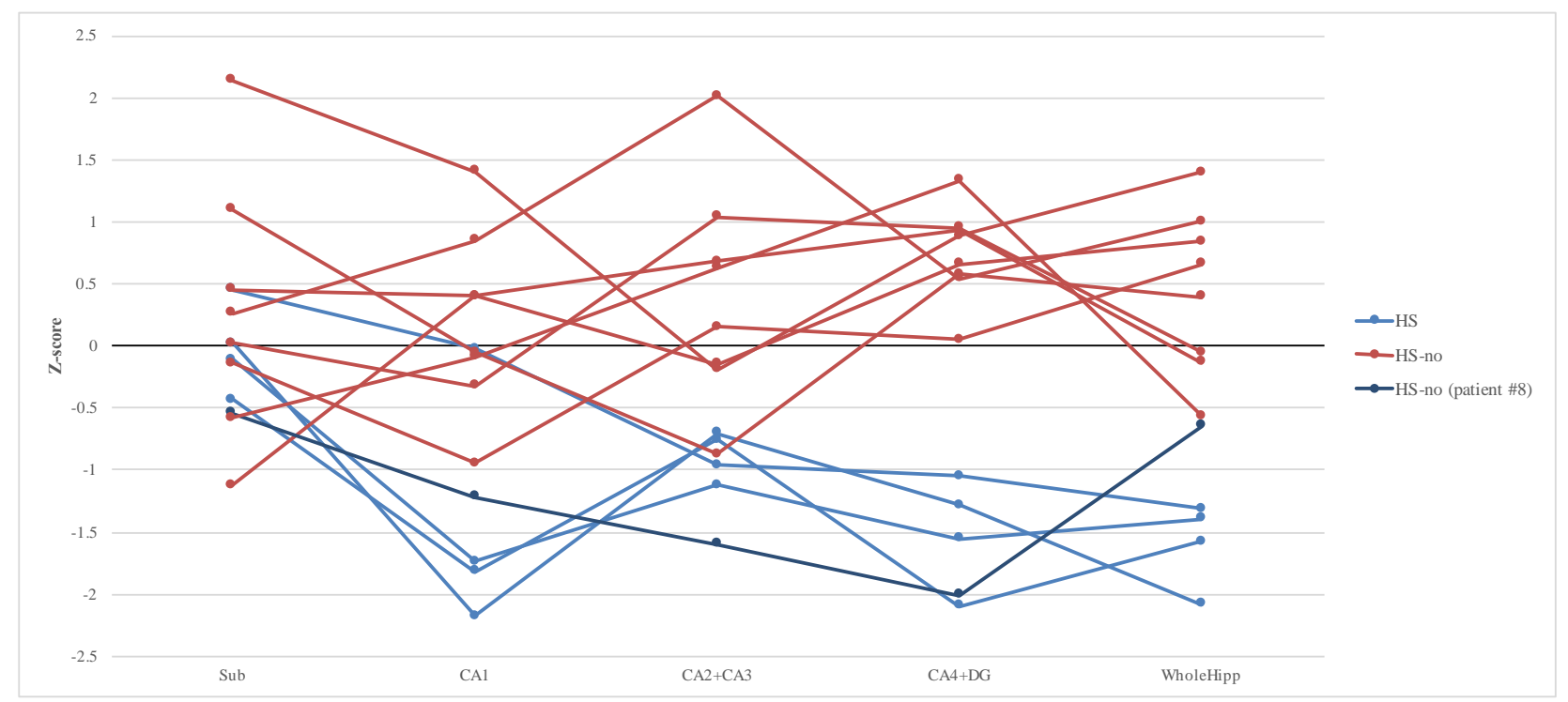

Figure 3: Line plots demonstrating subject-specific patterns of ipsilateral atrophy. Zscores were calculated and plotted with respect to each subfield for every patient and grouped by 1.5T MRI designation. The single HS-no patient (patient \# 8) demonstrating an atrophy pattern similar to those designated HS was grouped separately. Sub, subiculum; WholeHipp, whole hippocampus label. 
A
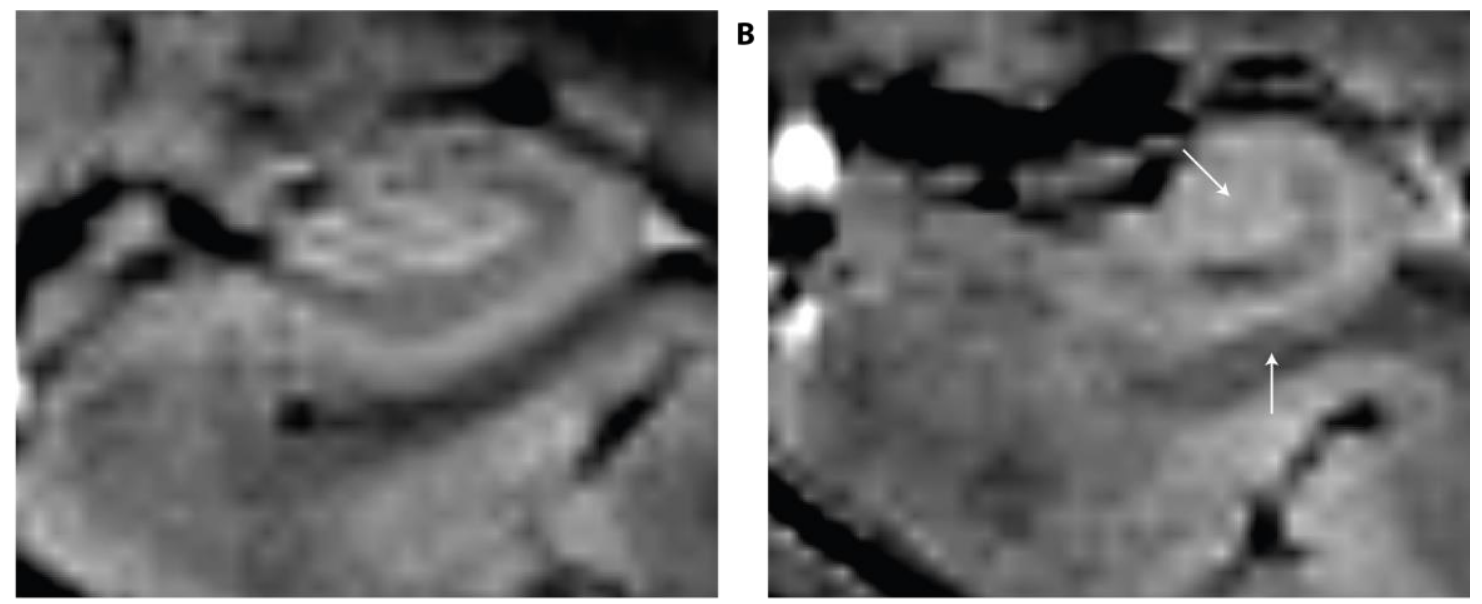

C

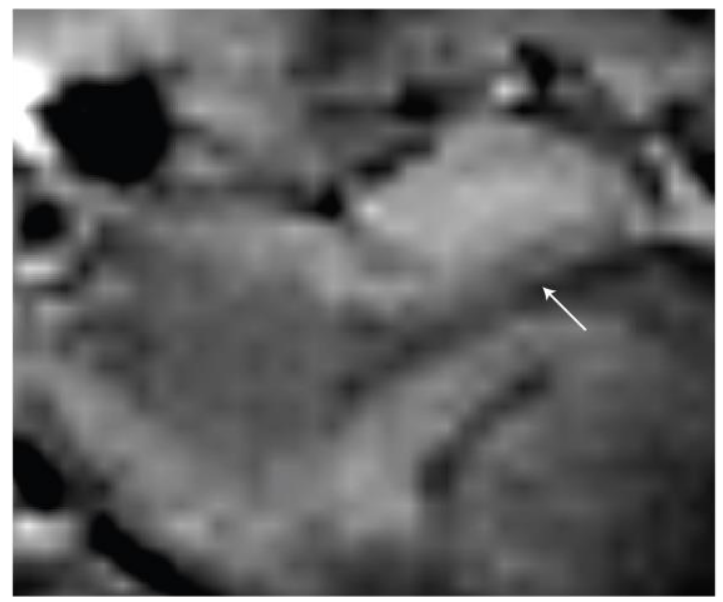

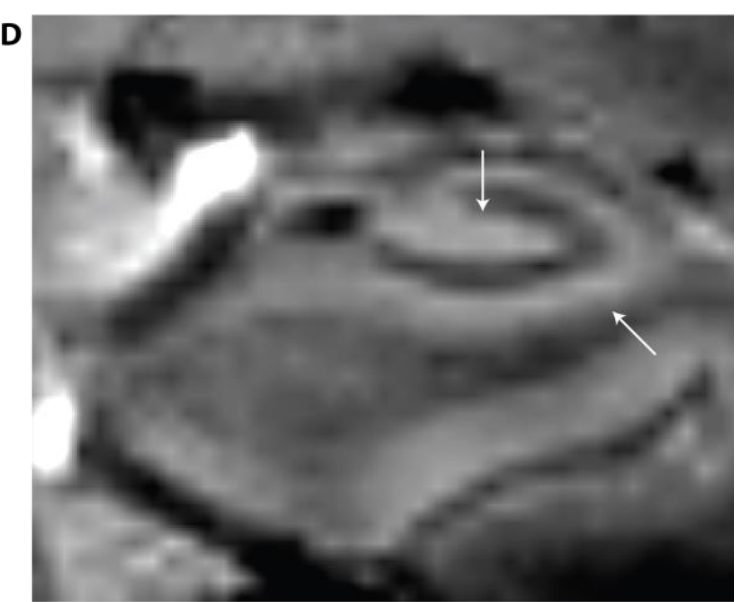

Figure 4: High resolution in vivo 7T T2-weighted representative coronal images of the hippocampal body demonstrating patterns of sclerotic change, with arrows indicating atrophy: (A) 33-year-old control. (B) 20-year-old HS patient with CA1 and CA4+DG volume loss and relative sparing of the other subfields. (C) 32-year-old HS patient with severe CA1 thinning and lesser CA4+DG atrophy with relative sparing of the other subfields. (D) 50-year-old HS-no patient with CA1 and CA4+DG volume loss (patient \#8). 